gescence of the veins of the eye and the lid, etc., are all due to an overfilling of the veins from regurgitation of blood from the sinus cavernosus, and he shows how this may readily occur, given a rupture of the artery, with consequent influx of arterial blood into the sinus with each pulsation of the heart. This tumor is simply an exposed vein, enlarged at this point and receiving in its contents an impulse with each influx of arterial blood into the sinus. Now, if there is a rupture of the artery within the sinus, the tumor, when it occurs, will always pulsate, a soft, compressible, pulsating tumor not communicating an impulse to the finger. Such a condition could not result from mere pressure, but must depend upon regurgitation. Only an inflow of arterial blood into the sinus can occasion pulsating regurgitation here.

As to the failure to find the gross appearances of a rupture of the artery at the post-mortem, that does not prove that there had been none. I doubt if an actual lesion of integrity will ever be found where as long a time has elapsed from the making of the operation to the making of the post-mortem section as had been the case here. There is ample time for a tear in the arterial coats to have healed perfectly, if at rest, in apposition, and no interference with nutrition in the immediate vicinity. Only an examination under a lens will disclose the scar there.

In regard to the hæmorrhage, it is impossible to say positively that it would have occurred if the patient had been entirely prudent. It is possible that, if undisturbed, a clot may have formed and organized. Certain it is that the great loss of blood, low. ering the general nutrition and acting on the area of brain already much lowered in vitality, assisted in the production of softening from malnutrition and thus brought on the fatal result.

\section{A CASE OF NEPHRECTOMY.}

Read before the Chicago Medical Society', Nov. 7, $18 S_{7}$. BY CHARLES T. PARKES, M.D.,

PROFESSOR OF SURGERY, RUSH MEDICAL COLIEGE, ATTENDING SLRGEON TO THE PRESBYTERIAN HOSPITAL, CHICAGO.

Last July I received a communication from a friend out of the city, stating that a patient had come under his charge who had been suffering for two years with cystitis, the diagnosis being based upon pus in the urine.

When I returned from my vacation in September, I found the patient awaiting me at one of the hospitals in the city. Upon examination it was found that a tumor could be easily palpated in the right side of the body beneath the ribs, large enough to extend down to the superior spinous fossa of the ileum, and reaching up to the hypogastric region below the liver. Upon the usual attempts at palpation and percussion, the dulness over the tumor was found to be continuous with the dulness of the liver. But the tumor appeared to me to be so elastic as to present some of the characteristics of a sac containing fluid. So I introduced an aspirator needle into it, and as was expected, found pus. As it presented none of the usual symptoms of a perinephritic abscess, it was diagnosticated to be a case of suppurative disease of the kidney communicating with the bladder through the ureter, the bladder being the outlet of the pus. There was no apparent disease of the bladder itself, other than that which would be present as a consequence of the foreign substance in the bladder.

Obtaining the patient's consent to an operation, an incision was made over the tumor to the outside of the erector spinæ muscle, and it was exposed; then the pockets of pus in the organ were located by the hypodermic syringe. On this occasion, three pockets of considerable size were opened, and drainage tubes introduced. About a pint of matter was let out. It was decided that these three pockets, that were found by introducing the syringe in different places, did not communicate with each other; they were separate cavities, and I think that is the usual condition found in this sort of disease of the kidneys; the pockets are multilocular.

One of them opened freely into the pelvis of the kidney, so that through the incision that was made the finger passed into the pelvis, and water injected into this went into the bladder, showing that there was a direct communication from this cavity of pus to the bladder and urethra. The drainage tubes were left in and the patient improved promptly, losing the fever and symptoms of pus accumulation and retention. For two weeks the improvement continued, then it was noticed that she began to fail rather rapidly and to show signs of fever again; there were signs of septic accumulation, and the tumor began to increase in size, so that from diminishing, perhaps half the size when first examined, it increased one third. As she was failing and the diagnosis was as complete as it was possible to make it, it was decided to perform nephrectomy.

There are some points of importance in the case: The drainage tube that went into the pelvis of the kidney gave free exit to quite a quanity of urine. I think that most of the secretion from that kidney came through the drainage tube; it was sufficient to wet thoroughly in two ur three hours a large dressing; this dressing was sufficient to keep the discharge from the wound pure so that there was nowhere decomposition of pus so far as the outward manifestations were concerned. It struck me that if this drainage tube from the diseased kidney gave exit to such an amount of urine, and at the same time there was a good flow of urine from the bladder, it was a fair indication that the other kidney was not diseased, and that success would attend the removal of the diseased kidney, and it was decided to do the operation.

Sixteen days ago the operation was done. The patient was prepared in a certain way that I have followed in reference to all patients upon whom I do what is considered a serious operation, and I think it has a certain influence in preventing shock. Two or three hours before the operation is performed the patient is given gr. $v$. to $\mathrm{x}$ of quinine, and gr. $\mathrm{I} / 4$ of morphine. This medicine was administered to the patient of whom I am speaking, and the opera- 
tion for the removal of the kidney was performed. The whole proceeding from beginning to end occupied an hour, and she went to bed without any manifestation of shock, and with a pulse of ir2. She had no rise of temperature until the second day, and then it rose to $100^{\circ}$, subsequent to that it fell to normal and did not rise above normal until the twelfth day, when other symptoms appeared. During all this time the wound was absolutely aseptic. It healed promptly by first intention, so that on the seventh day all stitches were removed; the wound was solid from one end to the other.

There are some points about this operation to which I desire to call your attention, and I will pass the specimen around to show the nature of the trouble. You will see at the lower end a cavity, which was found to contain six or eight ounces of pus; there is another cavity in the interior of the kidney, the pelvis is entirely destroyed and filled up with adventitious material.

Here was an operation to be done upon a mod. erately sized woman for the removal of a tumor containing pus, a tumor which reached up under the ribs, down to the crest of the ilium, and forward to the anterior spinous process. There was a tumor containing pus in which large pockets had formedwhat was the best way to remove it? There is no question in my mind that the best operation, in general, for the removal of the kidneys is the posterior operation. However, there are many diseases for which this operation is done where it is impossible to do it in another way than by the anterior operation, such as cases of cystic degeneration where the tumor is so large that it cannot be extruded posteriorly, but here was a tumor of moderate size, containing pus, in which it was desired above all things to avoid getting into the peritoneal cavity, a tumor which had sacs, the walls of which were in moderate degrees of thickness and strength, but could easily be broken upon pressure.

Therefore, the day before the operation I took a cadaver and experimented upon the lines of incision which would best expose this tumor and give exit to it. I finally decided upon the incisions represented in Figure $\mathbf{r}$. This represents the patient lying upon the opposite side from the diseased kidney. In these experiments $I$ found that by a certain incision I could get the amplest room without doing injury to the colon or peritoneum; certainly no more likely to injure the colon, the peritoneum or other contents of the abdominal cavity than in an operation for the exposure of any of the large blood-vessels of the abdomen.

It is hardly necessary for me to state that there is some little difference between subserous tissue in the lower portion of the abdomen and that of the upper. In the lower portion it is very loose and easily separated, whereas at the upper portion it is quite thin and the peritoneum is more apt to be torn, hence more care should be used in an operation in this position. The commencement of the incision is supposed to be two inches above the anterior superior spine of the ilium. It is carried in a curved direction downwards and backwards to the last rib. The incision is carried through all the tissues, down to the fascia transversalis, everything is carried forward out of the way, and with the finger the dissection can be made, well behind the tumor; all the parts are separated, then a straight incision is made through all of them, straight back from the first incision and half way between the crest of the ilium and the last rib. The introduction of a ligature at the point of the posterior flap, and pulling aside, there is a wound one can get both hands into, and by exposing the kidney in all its parts, reach the tissues which one wishes to have under control, without difficulty. Figure 2. In this case as soon as the

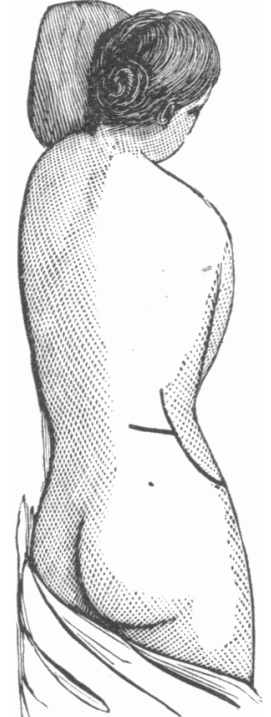

Figure 1,

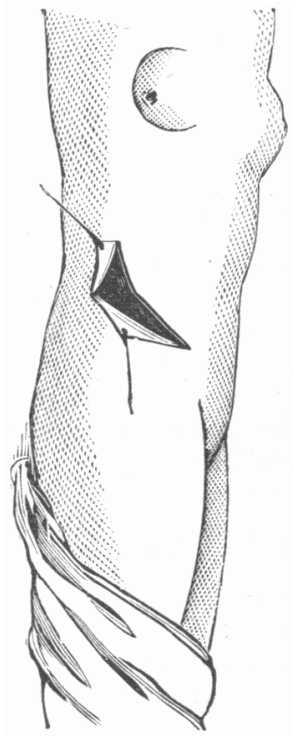

Figure 2. incision was made the tumor presented itself, the finger could be carried around it in all directions so that the kidney with its blood-vessels, ureter, and all could be exposed to view.

I desired to adopt the plan, which is a good one to adopt in all tumors that are difficult to reach, of diminishing the size of the tumor, and attempted to divide it in halves by the cautery, but after a few strokes of the knife I approached a pocket of pus, and gave that up for fear of infecting the wound. I had very little difficulty by taking an eyed probe, threaded with a stout double ligature, in passing the probe through the centre of the pedicle, and then with the double ligature ligating it in halves. The ligatures controlled the circulation perfectly. The vessels in the exposed stump were picked up and ligated one by one as a special security. Drainage was perfect, and the anterior wall of the peritoneum fell easily into place and united by primary intention. At the end of a week there was nothing left of this large cavity but the track of the drainage tube.

This case is an important illustration, it seems to me, of the safety of the posterior incision for tumors of considerable size, where the line of incision is carried out, somewhat in the way here indicated. The saddest part of my report is to come: Four days ago this patient was taken with symptoms of 
cerebral trouble and suppression of urine, and she died with all the symptoms of uræmia this afternoon at $\mathrm{r}$ o'clock, sixteen days after the operation. As yet a satisfactory examination has not been made to determine the condition of the opposite kidney, neither has there been a satisfactory microscopic examination of the tumor. Several sections have been submitted to examination, but none have shown tubercular bacillus.

The post-mortem examination showed a highly congested swollen organ in the remaining kidney, the capillary vessels were ruptured in many places.

\section{LOCAL MASSAGE FOR LOCAL NEURASTHENIA.}

Read before the Section for Clinical Medicine, Pathology, and Hygiene, of the Suffolk District Medical Socicty, November 9,1887 .

BY DOUGLAS GRAHAM, M.D., OF BOSTON, MASS.

Neurasthenia, as I understand it, may be either general or local, affecting the nerves or nerve-cells of all, or any part of the cerebro-spinal or sympathetic system. Its manifestations are those of exhaustion or too easy exhaustibility of nerve-force; and its pathology, malnutrition of the nerve cells, involved with concomitant instability of their circulation in the form of anæmia or hyperæmia, or alternations of these. It predisposes to, it accompanies, it results from disease; the nervous shock and the tedious recovery from injuries point to other.sources, and it may be caused by overwork, worry or sheer laziness. The agreeable fatigue after a satisfactory day's work, that insures sound sleep, may be regarded as a healthy form of neurasthenia, if the Hibernianism may be pardoned.

It is a matter of common observation, that those who are compelled to hard manual labor, seldom suffer from nervous prostration; and amongst the more fortunate who may be predisposed to neurasthenia, those who are deeply interested in some hobby or occupation that keeps mind and body active, have found the best means of prophylaxis. The same means that serves for its prevention, also supplies us with a clue to one of the most valuable agents that can be employed for its relief in recovery. Exercise keeps the circulation active, but requires effort of brain, spinal cord and nerves, as well as muscles, at a time when our object may be to afford rest to one or all of these parts of an overtaxed nervous system. Massage supplys this want, and will keep the circulation going with a minimum or no expenditure of nerve-force from the patient, and deep massage without friction will lessen the beats of the heart, and afford it rest also. Nay more, for it is getting to be the fashion not only amongst the laity, but also with some physicians, to say that massage imparts energy to the patient, though I confess I do not exactly understand what this means. Certainly, many who submit to massage feel much more vigorous, light, and supple after even the first application than they did before it. But may not this rather be owing to rousing of their latent energies, and restor- ing the equilibrium of their forces, by facilitating the circulation of blood, lymph, and the transmission of nerve-force?

I have previously stated elsewhere that in cerebral exhaustion the relative value of massage was almost nil, and that out-of door exercise was of paramount importance; but I have since found reason to modify this in favor of more massage and less exercise. In such cases, massage of the heac' alone daily, or every other day, is better than applying it all over the patient, unless there be a rare idiosyncrasy that will not allow the head to be manipulated.

There are people, not a few, who, when using their brains, suffer from uneasy sensations in the lumbar or dorsal region, and these discomforts continue after the cessation of study, causing wakefulness. Generally, there is also some spinal irritation in the region affected. In such cases, massage of the back alone will often induce sound sleep, and, next day, the patient feels inspired with faith, hope, and courage, in place of doubt, dread, and fear of meeting appointments. With these cases, a much more marked effect is produced by local than by general massage, except when the tenderness of the muscles and spinal irritation is extreme, unfitting them for every kind of work, and the massage should be general, omitting the back at the first séances, but gradually approaching it at subsequent ones.

In other cases of what may be called local neurasthenia, if the term can be allowed for this purpose, such as writer's cramp, or the cold, small, and feeble muscles resulting from injury, disease, or disuse, massage and exercise, carefully adapted, have given excellent results. To these have recently been added another affection; namely, laryngeal cramp of musicians and speakers, for the local treatment of which electricity and massage are considered the most effectual measures.

It is not the purpose of this paper to go into the details of applying massage, nor to consider its minute effects; but $I$ think it will be a revelation to many to experience either in their own heads, or to observe in those of their patients, the light, comfortable, delightful feelings that are produced by the resistance of a skilled manipulator to forward, backward, and lateral movements of the head. The impression is that the interior of the head has been benefited, and the effect is hardly secondary to massage, which rather gives the impression that the exterior has been improved.

The following cases seem to me sufficiently worthy of notice as examples of the conditions mentioned: Case 1.-A. J., 23 years of age. Three years prior to my being called to him, he had been running races at college at the same time that the functions of his brain flagged, and study had become so irksome, producing headache and insomnia, that he gave it up for a year. At the end of that time he returned to college for a year, and, to use his own words "patched up and graduated," and for the year before I saw him he had been trying to recuperate by resting at home. At this time, even walking sometimes produced discomfort in his head. At my first visit he had been suffering from headache, with tol. 